\title{
EFFECT OF DRIVING DURATION ON EEG FLUCTUATIONS
}

\author{
Maya Arlini Puspasari ${ }^{1 *}$, Hardianto Iridiastadi ${ }^{1}$, Iftikar Zahedi Sutalaksana ${ }^{1}$, Ade Sjafruddin ${ }^{2}$ \\ ${ }^{1}$ Faculty of Industrial Technology, Institut Teknologi Bandung, Jl. Ganesha No.10, Bandung 40132, \\ Indonesia \\ ${ }^{2}$ Faculty of Civil and Environmental Engineering, Institut Teknologi Bandung, Jl. Ganesha No.10, \\ Bandung 40132, Indonesia
}

(Received: February 2017 / Revised: May 2017 / Accepted: October 2017)

\begin{abstract}
Road accidents are a major issue in Indonesia, and their number increases every year. Based on previous studies, mental fatigue is one of the biggest factors leading to road accidents and is majorly affected by mental workload. Driving duration is one of the factors that triggers mental fatigue. The prior literature cites electroencephalogram (EEG) measurement as the gold standard for measuring fatigue. However, there has been only limited study to examine the EEG indicators that are affected by driving duration, and the prior research still contains disagreements regarding the best EEG parameter for use in measuring fatigue. Therefore, this study aimed to evaluate the effect of driving duration on EEG fluctuation and determine the best EEG parameter related to fatigue. Seven participants were asked to spend three hours driving in a medium-fidelity simulator. A one-way ANOVA and correlation analysis were performed to measure the effect of driving duration on the EEG indicators and determine the correlation of the indicators. A Receiver Operating Characteristics (ROC) curve was also utilized to determine the variable with the greatest correlation with the subjective sleepiness indices. The results showed that at the end of three hours' driving, there was an increment in delta and theta activities, followed by a decrement in alpha and beta activities. In addition, the correlation of all bands was significant, with positive results for the alpha-beta and theta-delta bands, and a negative result in relation to each other. Furthermore, the results from the ROC curve revealed the Relative Power Ratio (RPR) of theta, the RPR of alpha, and the ratio of $\theta / \alpha+\beta$ to be the best indicators among others, demonstrating a high degree of accuracy (above $85 \%)$.
\end{abstract}

Keywords: Driving Duration; EEG; Fatigue; Road Accident

\section{INTRODUCTION}

Fatigue is one of the fundamental factors contributing to road accidents. According to Zhang et al. (2013), fatigue contributes to $20 \%$ of road accidents, as it stimulates vigilance loss (Desai \& Haque, 2006) and performance decrement (Williamson et al., 2011). Previous research has settled on a definition of fatigue as a non-optimal psychophysiological condition that affects performance and is caused by time of day, homeostatic, and task-related factors (May \& Baldwin, 2009; Williamson et al., 2011; Phillips et al., 2015).

Driving duration is one of the factors that induces fatigue (Williamson et al., 2011; May \& Baldwin, 2009). Previous research has investigated the driving duration effect on physiological

\footnotetext{
*Corresponding author's email: mayaarlini@s.itb.ac.id, Tel: +62-22-2504551, Fax: +62-22-2509406

Permalink/DOI: https://doi.org/10.14716/ijtech.v8i6.716
} 
and performance decrement (Schleicher et al., 2008; Di Stasi et al., 2012; Wang \& Pei, 2014; Gastaldi et al., 2014). However, most of the prior research has studied the effect of short driving duration, which is defined as below two hours (Schleicher et al., 2008; Di Stasi et al., 2012; Gastaldi et al., 2014). Wang and Pei (2014) investigated the effect of four hours of driving on performance measures; however, a study looking at the possibility of using other indicators has not yet been conducted.

Electroencephalogram (EEG) has been accepted as the 'gold standard' of fatigue measurement in much of the prior literature (Jap et al., 2011; Jagannath \& Balasubramanian, 2014; Perrier et al., 2016). Previous studies have suggested that prolonged driving duration had varied effects on EEG fluctuations, consisting of disappearance of the alpha band with increments in theta and delta (Cajochen et al., 1995), increments in the alpha and theta bands (Jagannath \& Balasubramanian, 2014; Perrier et al., 2016), and a decrement in the beta band (Otmani et al., 2005). Furthermore, Jap et al. (2011) studied the effects of the monotony of train driving for 30 minutes and compared several parameters of EEG; $\theta / \beta, \theta /(\alpha+\beta),(\theta+\alpha) / \beta$, and $(\theta+\alpha) /(\alpha+\beta)$. Parameter $(\theta+\alpha) / \beta$ was concluded to be the best indicator of fatigue. Yet the aforementioned studies continued to show disagreement with regard to the best EEG parameter to measure fatigue. There is thus a need to conduct an in-depth study that focuses more on EEG indicators and driving duration as a factor that induces fatigue.

The novelty of this study is seen in how it addresses several parameters of EEG that are valid as measurements of driving fatigue, as the previous research still contained disagreements regarding this matter. Driving duration was used as an independent variable that induces fatigue. Thus, this study aimed to investigate the effect of a long driving duration on EEG fluctuation and determine the best EEG parameter to measure fatigue.

\section{METHODS}

Seven commercial drivers aged 25 to 35 years were involved in this study. They were asked to drive a medium-fidelity driving simulator for three hours. The driving simulator was a Logitech series with three pedals and embedded Citycar Driving software. This research used an electroencephalogram (EEG) headset (Emotiv, US) as the device to measure fatigue. The devices used in the research are shown in Figure 1.

The participants were prohibited from consuming caffeine and smoking prior to the experiment. They had slept for a minimum of seven hours prior to the experiment and their activity levels were measured using an activity tracker (Fitbit Charge HR, US). They consumed meals provided by the researcher on the day of the experiment.

Emotiv EEG was measured before and after the driving task. The measurement points were six frontal channels (F3, F4, F7, F8, AF3, and AF4) out of a total of 16 channels (as shown in Figure 2). This brain measurement followed previous studies (Otmani et al., 2005; Jagannath \& Balasubramanian, 2014; Perrier et al., 2016), which stated that the frontal part of the brain controls cognitive skill and is affected by fatigue.

EEG data were taken for a period of five minutes before and after driving and these were then aggregated into one minute each for advanced measures, based on Gillberg et al. (1996). The EEG data processing was conducted as per the five steps below:

1) Pre-processing (Band Pass Filter) to eliminate the noise of outside brain frequency signal (below $0.1 \mathrm{~Hz}$ and above $25 \mathrm{~Hz}$, based on Yeo et al. (2009))

2) Signal decomposition (Fast Fourier Transform), which decomposited the brain waves based on their frequency (delta for $0-4 \mathrm{~Hz}$, theta for 4-7 Hz, alpha for 7-13 Hz, and beta for 13-20 Hz), based on Tatum (2014) 
3) Power Spectral Density processing that measures the power of brain waves (in dB unit)

4) Formulation of the Relative Power Ratio (RPR) to determine the variables of alpha, beta, theta, and delta, using the following formula:

$$
\text { Relative Power Ratio }=\left(\frac{\text { PowerOfEachBand }}{\text { PowerOfAllBand }}\right) \times 100
$$

5) Determine the ratio of the EEG parameters $(\theta / \beta, \theta /(\alpha+\beta),(\theta+\alpha) / \beta$, and $(\theta+\alpha) /(\alpha+\beta))$.
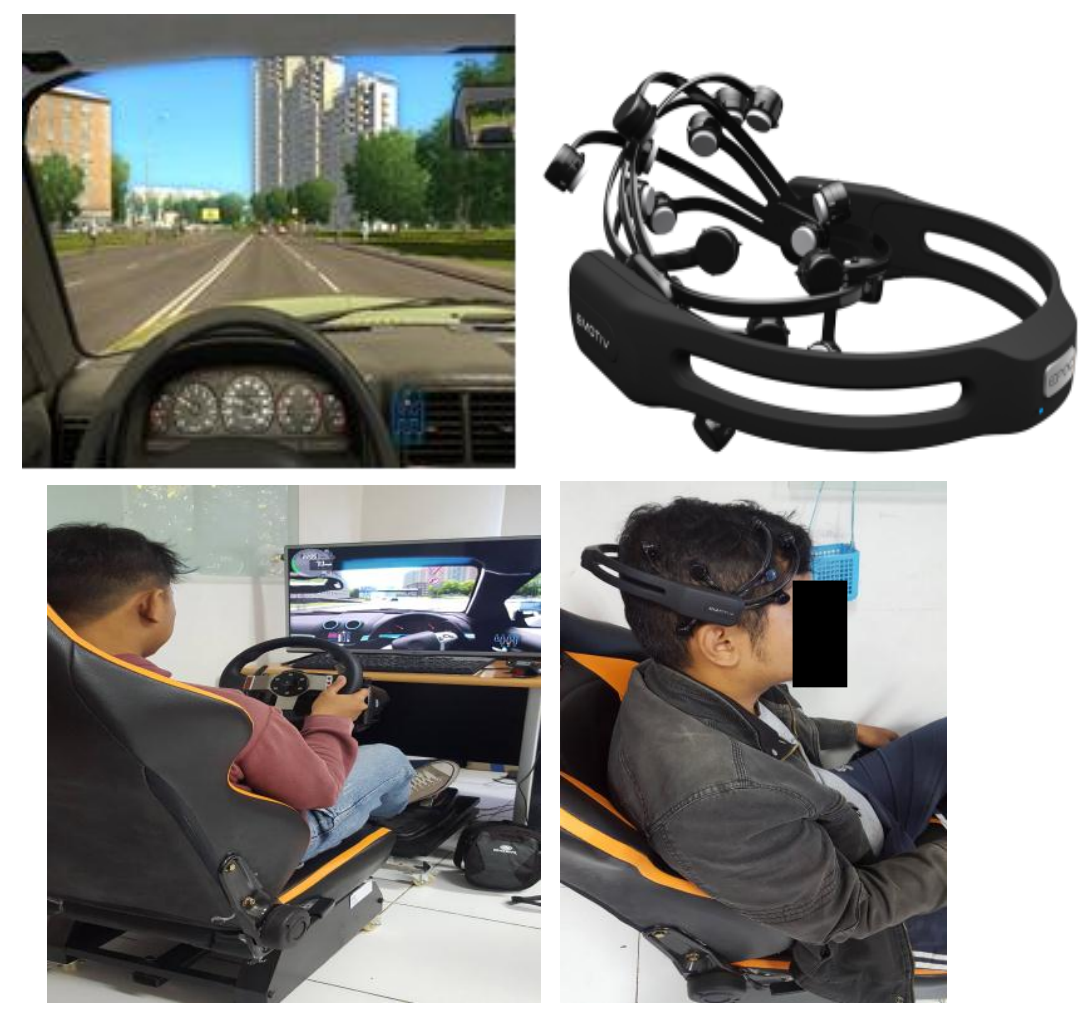

Figure 1 The devices used in this research

(Source: emotiv.com, citycardriving.com, and photos taken during the experiment)

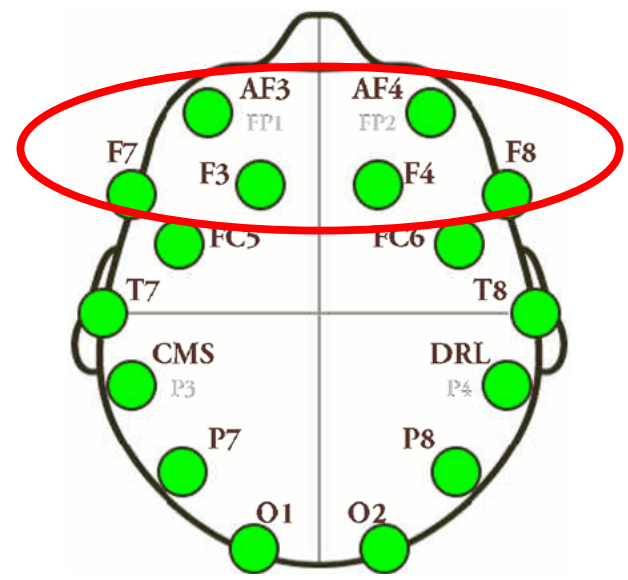

Figure 2 The six measurement points used in this research 
Besides using EEG measurement as an objective indicator, this study also employed a subjective scale as a reference point for fatigue measurement. The Karolinska Sleepiness Scale, which consists of a 9-point scale, was used in this study based on its validity as stated in several previous studies (Kaida et al., 2006; Abe et al., 2011; Jagannath \& Balasubramanian, 2014).

A one-way ANOVA with the driving duration factor was performed on each EEG indicator, along with correlation analysis. A Receiver Operating Characteristics (ROC) curve was also utilized to determine the best parameter to measure fatigue, along with the sensitivity and specificity of each parameter (Abe et al., 2011). A comparison of each band was also discussed along with the previous literature.

\section{RESULTS AND DISCUSSION}

\subsection{Results}

The descriptive statistics for RPR in each band (beta, alpha, theta, and delta) and all ratios $(\theta / \beta$, $\theta /(\alpha+\beta),(\theta+\alpha) / \beta$, and $(\theta+\alpha) /(\alpha+\beta))$ can be seen in Table 1 .

Table 1 Descriptive statistics for RPR and ratios in the beta, alpha, theta, and sdelta bands

\begin{tabular}{lcc}
\hline \multicolumn{1}{c}{ Variables } & Before Driving & After Driving \\
\hline RPR alpha & $0.239 \pm 0.003$ & $0.232 \pm 0.010$ \\
RPR beta & $0.289 \pm 0.004$ & $0.266 \pm 0.041$ \\
RPR theta & $0.222 \pm 0.002$ & $0.228 \pm 0.010$ \\
RPR delta & $0.248 \pm 0.006$ & $0.271 \pm 0.041$ \\
$\theta / \beta$ & $0.769 \pm 0.019$ & $0.895 \pm 0.244$ \\
$\theta /(\alpha+\beta)$ & $0.421 \pm 0.009$ & $0.467 \pm 0.085$ \\
$(\theta+\alpha) / \beta$ & $1.595 \pm 0.022$ & $1.788 \pm 0.379$ \\
$(\theta+\alpha) /(\alpha+\beta)$ & $0.873 \pm 0.010$ & $0.936 \pm 0.119$ \\
\hline
\end{tabular}

From Table 1, the alpha band decreased by $2.3 \%$ in period 2 (after driving) compared to before driving. The beta band also decreased by $6.8 \%$ in period 2 . However, there were increments in the theta and delta bands of $2.1 \%$ and $8.2 \%$, respectively. All of the ratios of $\theta / \beta, \theta /(\alpha+\beta)$, $(\theta+\alpha) / \beta$, and $(\theta+\alpha) /(\alpha+\beta)$ increased after driving by around 7 to $16 \%$, suggesting the influence of the theta, alpha, and beta bands on fatigue. All of the measured parameters showed significant changes in relation to driving duration. The statistical results are shown in Table 2.

Table 2 Statistical results of RPR

\begin{tabular}{lcc}
\hline \multicolumn{1}{c}{ Variables } & $\mathrm{F}$ & $p$-value \\
\hline RPR Alpha & 6.94 & 0.011 \\
RPR Beta & 6.06 & $\mathbf{0 . 0 1 7}$ \\
RPR Theta & 6.59 & $\mathbf{0 . 0 1 3}$ \\
RPR Delta & 6.17 & $\mathbf{0 . 0 1 7}$ \\
$\theta / \beta$ & 5.29 & $\mathbf{0 . 0 2 6}$ \\
$\theta /(\alpha+\beta)$ & 5.70 & $\mathbf{0 . 0 2 1}$ \\
$(\theta+\alpha) / \beta$ & 5.17 & $\mathbf{0 . 0 2 7}$ \\
$(\theta+\alpha) /(\alpha+\beta)$ & 5.55 & $\mathbf{0 . 0 2 3}$ \\
\hline
\end{tabular}

The statistical results show that all bands and ratios had a significant difference from before to after driving. Correlation analysis (Pearson-p) also revealed all bands to have significant correlation, with alpha-beta and theta-delta having positive correlation (Pearson-p of 0.989 and 
0.994, respectively), and alpha-theta, alpha-delta, beta-theta, and beta-delta displaying negative correlation (Pearson-p of $-0.993,-0.992,-0.995$, and -1.000 , respectively).

Moreover, Karolinska Sleepiness Scale (KSS) was set as the reference point of fatigue, which was divided into two categories: alert condition (KSS 1-6) and fatigue condition (KSS 7-9), in line with Pauly and Shankar (2015). The EEG data were then mapped onto the reference points and analyzed using the ROC curve. The cutoff point, accuracy, sensitivity, and specificity were obtained for each of the parameters, as shown in Table 3.

Table 3 Results from receiver operating characteristics

\begin{tabular}{lccccc}
\hline \multicolumn{1}{c}{ Variables } & AUC & $p$ & Cutoff value & Sensitivity & Specificity \\
\hline RPR Alpha & $89.4 \%$ & $<0.001$ & 0.238 & $96 \%$ & $64 \%$ \\
RPR Beta & $85.9 \%$ & $<0.001$ & 0.286 & $88 \%$ & $76 \%$ \\
RPR Theta & $91.2 \%$ & $<0.001$ & 0.223 & $88 \%$ & $88 \%$ \\
RPR Delta & $85.6 \%$ & $<0.001$ & 0.251 & $92 \%$ & $76 \%$ \\
$\theta / \beta$ & $86.7 \%$ & $<0.001$ & 0.779 & $92 \%$ & $76 \%$ \\
$\theta /(\alpha+\beta)$ & $89 \%$ & $<0.001$ & 0.426 & $92 \%$ & $80 \%$ \\
$(\theta+\alpha) / \beta$ & $86.4 \%$ & $<0.001$ & 1.604 & $96 \%$ & $72 \%$ \\
$(\theta+\alpha) /(\alpha+\beta)$ & $86.7 \%$ & $<0.001$ & 0.879 & $92 \%$ & $76 \%$ \\
\hline
\end{tabular}

The results in Table 3 reveal RPR theta, RPR alpha, and $\theta /(\alpha+\beta)$ to be the three parameters with the highest AUC scores. These three parameters also demonstrated good sensitivity and specificity (from 64 to 96\%). It can thus be concluded that the participants were classified as being in a fatigued state if they had an RPR theta in excess of 0.223 , RPR alpha higher than 0.238 , and $\theta /(\alpha+\beta)$ greater than 0.426 . The ROC curve for RPR theta can be seen in Figure 3 .

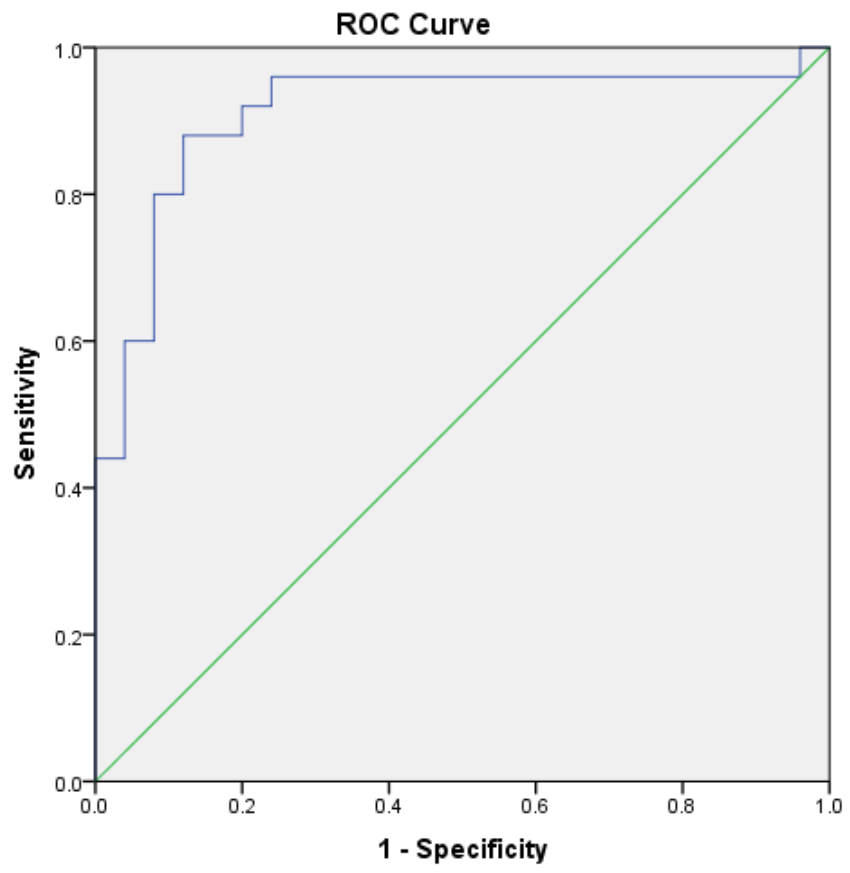

Figure 3 ROC curve of RPR theta

This study utilized seven participants and had a sufficient sample size. The power of the experiment was calculated using effect size d (Maxwell \& Delaney, 2003). The mean and 
standard deviation of all of the average parameters of EEG before and after driving were compared using formula 2 .

$$
d=\frac{\mu_{\max }-\mu_{\min }}{\sigma_{s}}
$$

The value of effect size $d$ for this data was 1.65. According to the table, the minimum sample size required to run the experiment with $\beta=0.5$ and two levels is above five participants. It can thus be concluded that this study had a sufficient sample size.

\subsection{Hydrodynamic Performance}

The aim of this study was to investigate the effects of long driving duration on EEG fluctuation and to determine the best EEG parameter to measure fatigue. In general, this study shows a promising result that complements the previous research.

The major findings of this study consist of two parts: fluctuation of EEG and best EEG parameter. The results showed an alpha-beta band decrement and theta-delta increment in line with fatigue onset, and this was consistent for all of the participants. The result is slightly different from those achieved by Jagannath and Balasubramanian (2014) and Perrier et al. (2016), whose results showed an increment in the alpha-theta band for fatigue. However, the result is in accordance with Cajochen et al. (1995), who stated that there is an increment in the theta-delta band as an indication of fatigue, and Otmani et al. (2005), who found a decrement in the beta band. On the other hand, the ratio of the EEG parameters $(\theta / \beta, \theta /(\alpha+\beta),(\theta+\alpha) / \beta$, and $(\theta+\alpha) /(\alpha+\beta))$ were all significantly affected by driving duration, with the after-driving period having a higher ratio than the before-driving period. This shows that the alpha, beta, and theta bands have a major effect in terms of their ability to indicate fatigue onset, which is in line with the results of previous studies (Otmani et al., 2005; Cajochen et al., 1995; Jagannath \& Balasubramanian, 2014; Perrier et al., 2016).

The results of this study showed that the RPR value of the theta band may present a robust indicator for detecting fatigue, as shown by the highest accuracy from the ROC curve, followed by RPR alpha and $\theta /(\alpha+\beta)$. These results complement those from previous research by Jap et al. (2011) that stated a ratio of $(\theta+\alpha) / \beta$ as the best indicator of detecting fatigue.

The implication of this research is related to fatigue onset. Driving for three hours increases a driver's subjective sleepiness from an alert to a fatigue condition (one participant experienced heavy fatigue, five participants experienced fatigue, and one participant experienced slight fatigue). It can thus be concluded that driving for three hours gives rise to varying fatigue conditions. A range of countermeasures are therefore needed, such as providing resting time and developing driving interventions.

The practical implications of this study relate to transport safety in general. EEG signals, particularly RPR theta, can be employed as a practical parameter for detecting fatigue when driving. Technology utilizing EEG signals could thus be developed to contain a fatigue detection function using RPR theta and fatigue interventions using visual, auditory, or haptic countermeasures.

The limitation of this study mainly relates to the instruments used. This study utilized a medium-fidelity driving simulator as the instrument to simulate real driving conditions. Based on Meuleners and Fraser (2015), the behavior of participants driving in a simulator is not significantly different from that seen in real driving situations. Davenne et al. (2012) stated that driving for four hours leads to a significant decrease in performance, yet this does not extend to subjective fatigue. It can thus be concluded that the driving simulator has relative validity that 
represents real driving. This study also suggests that real driving or a high-fidelity simulator can be utilized in future research to obtain better results and external validity.

\section{CONCLUSION}

This study aimed to evaluate the effects of driving duration on EEG fluctuation and determine the best EEG parameter related to fatigue. This research used three hours of driving in a medium-fidelity driving simulator and measured EEG before and after the experiment. The results show that the alpha, beta, theta, and delta bands have a significant difference from before to after driving, with an increment in the theta-delta band and a decrement in the alphabeta band. The correlations of all bands show significant results, with alpha-beta and theta-delta having a positive correlation, and alpha-theta, alpha-delta, beta-theta, and beta-delta having a negative correlation. Furthermore, the results from the ROC curve reveal the RPR of theta, RPR of alpha, and the ratio of $\theta / \alpha+\beta$ to be the best indicators among others, with accuracies of $91.2 \%, 89.4 \%$, and $89 \%$, respectively.

\section{ACKNOWLEDGEMENT}

The authors would like to thank the Work System and Ergonomics Laboratory of Institut Teknologi Bandung for funding this experiment and the Ergonomics Centre Universitas Indonesia for the device used in this experiment.

\section{REFERENCES}

Abe, T., Nonomura, T., Komada, Y., Asaoka, S., Sasai, T., Ueno, A., Inoue, Y., 2011. Detecting Deteriorated Vigilance using Percentage of Eyelid Closure Time during Behavioral Maintenance of Wakefulness Tests. International Journal of Psychophysiology, Volume 82, pp. 269-274

Cajochen, C., Brunner, D.P., Krauchi, K., Graw, P., Wirz-Justice, A., 1995. Power Density in Theta/Alpha Frequencies of the Waking EEG Progressively Increases during Sustained Wakefulness. Sleep, Volume 18, pp. 890-894

Davenne, D., Lericollais, R., Sagaspe, P., Taillard, J., Gauthiera, A., Espiéc, S., Philip, P., 2012. Reliability of Simulator Driving Tool for Evaluation of Sleepiness, Fatigue and Driving Performance. Accident Analysis and Prevention, Volume 45, pp. 677-682

Desai, A.V., Haque, M.A., 2006. Vigilance Monitoring for Operator Safety: A Simulation Study on Highway Driving. Journal of Safety Research, Volume 37, pp. 139-147

Di Stasi, L.L., Renner, R., Catena, A., Canas, J.J., Velichkovsky, B.M., Pannasch, S., 2012. Towards a Driver Fatigue Test based on the Saccadic Main Sequence: A Partial Validation by Subjective Report Data. Transportation Research Part C, Volume 21, pp. 122-133

Gastaldi, M., Rossi, R., Gecchele, G., 2014. Effects of Driver Task Related Fatigue on Driving Performance. Procedia - Social and Behavioral Sciences, Volume 111, pp. 955-964

Gillberg, M., Kecklund, G., Akerstedt, T., 1996. Sleepiness and Performance of Professional Drivers in a Truck Simulator-Comparisons between Day and Night Driving. Journal of Sleep Research, Volume 5, pp. 12-15

Jagannath, M., Balasubramanian, V., 2014. Assessment of Early Onset of Driver Fatigue using Multimodal Fatigue Measures in a Static Simulator. Applied Ergonomics, Volume 45, pp. 1140-1147

Jap, B.D., Lal, S., Fischer, P., 2011. Comparing Combinations of EEG Activity in Train Drivers during Monotonous Driving. Expert System with Applications, Volume 38, pp. 996-1003

Kaida, K., Takahashi, M., Akerstedt, T., Nakata, A., Otsuka, Y., Haratani, T., Fukasawa, K., 2006. Validation of the Karolinska Sleepiness Scale against Performance and EEG Variables. Clinical Neurophysiology, Volume 117, pp. 1574-1581 
Maxwell, S.E., Delaney, H.D., 2004. Designing Experiments and Analyzing Data a Model Comparison Perspective ( $2^{\text {nd }}$ edition). Lawrence Erlbaum Associates, New Jersey

May, J., Baldwin, C.L., 2009. Driver Fatigue: The Importance of Identifying Causal Factors of Fatigue when Considering Detection and Countermeasure Technologies. Transportation Research Part F, Volume 12, pp. 218-224

Meuleners, L., Fraser, M., 2015. A Validation Study of Driving Errors using a Driving Simulator. Transportation Research Part F, Volume 29, pp. 14-21

Otmani, S. Pebayle, R., Muzet., 2005. Effect of Driving Duration and Partial Sleep Deprivation on Subsequent Alertness and Performance of Car Drivers. Physiology \& Behavior, Volume 84, pp. 715-724

Pauly, L., Shankar, D., 2015. Detection of Drowsiness based on HOG Features and SVM Classifiers. Proceedings of IEEE International Conference on Computer Graphics, Vision, and Information Security (CGVIS)

Perrier, J., Jongen, S., Vuurman, E., Bocca, M.L., Ramaekers, J.G., Vermeeren, A., 2016. Driving Performance and EEG Fluctuations during on-the-road Driving Following Sleep Deprivation. Biological Physiology, Volume 12, pp. 1-11

Phillips, R.O., 2015. A Review of Definitions of Fatigue - And a Step towards a Whole Definition. Transportation Research Part F, Volume 29, pp. 48-56

Schleicher, R., Galley, N., Briest, S., Galley, L., 2008. Blinks and Saccades as Indicators of Fatigue in Sleepiness Warnings: Looking Tired?. Ergonomics, Volume 51, pp. 982-1010

Tatum, W.O, 2014. Handbook of EEG Interpretation (2 ${ }^{\text {nd }}$ edition). Demos Medical Publishing, New York

Wang, L., Pei, Y., 2014. The Impact of Continuous Driving Time and Rest Time on Commercial Drivers' Driving Performance and Recovery. Journal of Safety Research, Volume 50, pp. 11-15

Williamson, A., Lombardi, D.A., Folkard, S., Stutts, J., Courtney, T.K., Connor, J.L., 2011. The Link between Fatigue and Safety. Accident Analysis \& Prevention, Volume 43, pp. 498-515

Yeo, M.V.M., Li, X., Shen, K., Wilder-Smith, E.P.V., 2009. Can SVM be Used for Automatic EEG Detection of Drowsiness during Car Driving. Safety Science, Volume 47, pp. 115-124

Zhang, G., Yau, K., Chen, G., 2013. Risk Factors Associated with Traffic Violations and Accident Severity in China. Accident Analysis \& Prevention, Volume 59, pp. 18-25 